\title{
Tratamiento quirúrgico de la hemorragia intracerebral espontánea. Parte I: Hemorragia supratentorial
}

\author{
A. Pérez-Núñez; A. Lagares; B. Pascual; J.J. Rivas; R. Alday; P. González; A. Cabrera y R.D. Lobato
}

Servicio de Neurocirugía. Hospital Universitario Doce de Octubre. Madrid.

Resumen

La hemorragia intracerebral espontánea (HIE) constituye uno de los procesos ictales de mayor gravedad. A pesar de esto y de una elevada incidencia, su tratamiento médico no va mucho más allá de un papel de soporte vital y control médico de la hipertensión intracraneal, y las indicaciones del tratamiento quirúrgico están pobremente basadas en evidencia científica.

El objetivo del presente trabajo fue revisar las bases de la indicación quirúrgica en la HIE supratentorial.

Encontramos 10 ensayos clínicos y 5 meta-análisis en lengua inglesa que analizaban la utilidad del tratamiento quirúrgico en esta patología. Aunque globalmente estos estudios no mostraron un beneficio significativo del tratamiento quirúrgico en el conjunto de pacientes con $\mathrm{HIE}$ supratentorial, existe un subgrupo de pacientes en los que parece que dicho tratamiento podría ser beneficioso.

En la hemorragia intracerebral espontánea supratentorial las recomendaciones actuales indican que los pacientes jóvenes, con hematomas lobares cuyo volumen causa un deterioro del nivel de consciencia, deben ser intervenidos. En pacientes con hematomas putaminales que reúnen las mismas condiciones de edad y deterioro neurológico la cirugía podría mejorar la evolución, al menos en términos de supervivencia. Un grave deterioro neurológico con $\mathbf{G C S}<\mathbf{5}$, la localización talámica y la presencia de una situación basal o edad que impidan una adecuada recuperación funcional, son criterios considerados tradicionalmente contraindicación del tratamiento quirúrgico. Dada la ausencia de evidencia científica sólida en la que sustentar estas recomendaciones, la decisión terapéutica debe realizarse de manera individualizada y prestando atención al soporte sociofamiliar del paciente, que jugará un papel importante en la evolución del mismo a medio/largo plazo.

PALABRAS CLAVE: Hemorragia intracerebral. Cirugía. Tratamiento.

Recibido: 25-07-07. Aceptado 1-10-07
Surgical treatment for spontaneous intracerebral haemorrhage. Part I: Supratentorial haematomas

Summary

Spontaneous intracerebral haematoma (SICH) represents one the most severe subtypes of ictus. However, and despite a high incidence, medical treatment is almost limited to life support and to control intracranial hypertension and indications of surgical treatment are poorly defined.

The aim of this paper was to review the evidence supporting surgical evacuation of SICH.

Ten clinical trials and five meta-analyses studying the results of surgical treatment on this pathology were found on English literature. These studies considered all together, failed to show a significant benefit of surgical evacuation in patients with SICH considered as a whole. However, a subgroup of these patients has been considered to potentially present a better outcome after surgical treatment.

Current recommendations on supratentorial intracerebral haemorrhage state that young patients with lobar haematomas causing deterioration on the level of consciousness should be operated on. Patients suffering from putaminal haematomas and fitting with the same criteria of age and neurological deterioration could also benefit from surgery, at least on terms of survival. Deep neurological deterioration with $\mathrm{GCS}<5$, thalamic location, severe functional deterioration on basal condition or advanced age precluding an adequate functional outcome, have been traditionally considered criteria contraindicating surgery. Given the absence of strong scientific evidence to indicate surgery, this measure should be taken on a tailored manner, and taking into account the social-familiar environment of the patient, that will strongly condition his/her future quality of life.

KEY WORDS: Intracerebral haemorrhage. Surgery. Treatment. 


\section{Introducción}

Los accidentes cerebrovasculares representan la tercera causa de muerte en el adulto, y un $10-15 \%$ de ellos corresponden a la hemorragia intracerebral espontánea (HIE), si bien esta proporción varía en función de la raza, elevándose al $20-30 \%$ en Japón y algunos países europeos $^{5,9,27,28,30,35}$. Su incidencia, que se ha estimado en 10-20 casos/100.000 habitantes año, aumenta con la edad, y así dos tercios de las HIE ocurren entre los 45 y 75 años y más de la mitad entre los 55 y los $75^{35}$. La hemorragia intracerebral espontánea (HIE) se define como aquélla no relacionada con un antecedente traumático o quirúrgico, y se divide en primaria (el $70-80 \%$ de los casos) o secundaria en función de la ausencia o presencia a corto plazo de un agente causal (tabla 1) ) $^{5,9,10,35,37}$. Aproximadamente un $70 \%$ de las HIE se asocian con hipertensión arterial, el principal factor de riesgo modificable, con un Riesgo Relativo (RR) entre 3.9 y $13.3^{9}$. La incidencia en el hombre es igual o ligeramente más alta que en la mujer, y es significativamente más elevada en las razas asiática y negra (en esta última, posiblemente debido a una mayor incidencia de hipertensión $)^{5,9,35}$. La angiopatía amiloide, y los genotipos $\varepsilon 2$ y $\varepsilon 4$ de la apolipoproteína E (relacionados con el depósito de dicho material en los vasos cerebrales) son también factores de riesgo, especialmente en pacientes ancianos $^{5,35}$. El consumo reciente de cantidades excesivas de alcohol, la diabetes mellitus, la hipocolesterolemia y el tratamiento anticoagulante también se han relacionado con el riesgo de sufrir una $\mathrm{HIE}^{5,9,10,35}$.

La mortalidad asociada a corto plazo alcanza el 50\% (23-58\%) y más de dos tercios de los supervivientes desarrollan algún grado de incapacidad permanente; de hecho, únicamente un $38 \%$ de los afectados sobreviven al año del episodio y sólo un 20\% viven independientes a los 6 meses $^{5,9,21,35,40}$. La edad avanzada, la situación funcional previa, la localización (fundamentalmente en tronco de encéfalo y también en tálamo o ganglios basales), el volumen del hematoma ( $>40-60 \mathrm{cc}$ ), la presencia de hemorragia intraventricular, la anticoagulación y un grado avanzado de deterioro neurológico son los factores pronósticos más adversos, resultando este último el más determinante cuando es considerado aisladamente ${ }^{3,9,10,27,28,31,38}$. Desde el punto de vista etiopatogénico la HIE hipertensiva se relaciona con daño de pequeñas arterias y arteriolas perforantes a causa de lesiones en el endotelio y la capa media, originando en ocasiones deformidades de la pared identificadas como microaneurismas por algunos autores ("miliary aneurysms" descritos por Charcot y Bouchard en las paredes de los vasos de los pacientes con hematomas hipertensivos), y la producción de la llamada angiopatía fibrinoide e hialinizante hipertensiva ${ }^{27,35}$. Zülch encontró degeneración de la pared vascular consistente en cambios arterioescleró-
Tabla 1

Causas de hemorragia intracerebral espontánea (HIE)

Causas de HIE primaria y secundaria

$$
\begin{aligned}
& \text { Primaria } \\
& \quad \text { - hipertensión } \\
& \text { - angiopatía amiloide }
\end{aligned}
$$

\section{Secundaria}

- aneurismas

- malformaciones vasculares

- neoplasias

- coagulopatía

- alcohol o drogas

- infarto cerebral con transformación hemorrágica

- trombosis de senos venosos durales

- vasculitis/vasculopatía

- enfermedad de moyamoya

- disección arterial

- embarazo (eclampsia, trombosis venosas)

- otras/criptogénica

ticos hialinizantes y microaneurismas en la virtual totalidad de las necropsias de HIE hipertensiva. El desarrollo de los sangrados parece sin embargo en relación con rotura de los vasos en la vecindad de las bifurcaciones más que con la rotura de los microaneurismas, que podrían representar fundamentalmente el daño de base sobre la pared de las arterias o la presencia de microsangrados en el espesor de la misma ${ }^{9,35}$. Estos cambios ocurren no sólo afectando a las arterias lentículo- y tálamo-estriadas, sino también a las de la sustancia blanca subcortical, el tronco y el cerebelo. La localización de la HIE hipertensiva refleja la afección de estos vasos, y así es más frecuente en los ganglios basales, especialmente el putamen, y en la zona de las cápsulas interna y externa. El $80 \%$ de los hematomas asientan por encima del tentorio y un $20 \%$ a nivel infratentorial (en el límite mesencéfalo-protuberancial y en la proximidad de los núcleos dentados del cerebelo). Jellinger registró las siguientes localizaciones preferentes en 11 series de HIE hipertensiva: $64 \%$ en los ganglios basales, $13 \%$ en sustancia blanca subcortical, $10-12 \%$ en tronco, $12 \%$ en cerebelo y $11 \%$ en tálamo. La incidencia de hemorragia intraventricular (HIV) asociada fue del $60 \%{ }^{30}$. El depósito de $\beta$-amiloide tiene lugar predominantemente en los vasos corticales y leptomeníngeos, y así los hematomas del 
anciano en relación con esta patología asientan fundamentalmente en los hemisferios cerebrales ${ }^{9,35}$.

En cuanto a la fisiopatología los factores responsables del deterioro neurológico son la disrupción del tejido vecino, el efecto masa local del hematoma (que produce compresión, isquemia y edema perilesionales) y el efecto masa generalizado que eleva la presión intracraneal (con o sin hidrocefalia asociada) y puede causar herniación cerebral $^{17,18,20,25,29,36}$. En un estudio necrópsico Freitag observó signos de compresión secundaria del tronco, que consideró responsable de la muerte, en la gran mayoría de los casos de hematoma de ganglios basales ${ }^{30}$. A pesar del gran número de estudios experimentales sobre la fisiopatología de la HIE, en los que se han utilizado fundamentalmente dos modelos (inyección estereotáxica de volúmenes variables de sangre, e hinchado de balones en la región de los ganglios basales), no se tiene una idea clara sobre la importancia relativa del daño neurológico primario o directo producido en el momento del sangrado (compresióndisrupción tisular), ni del secundario debido a la isquemia (disminución del flujo cerebral) y el edema (citotóxico o vasogénico) perifocales, o al posible daño tóxico inducido por los productos de degradación del hematoma ${ }^{9,23,35}$.

La clínica típica de la HIE se caracteriza por la aparición brusca de déficit neurológico acompañado de cefalea, náusea, vómitos, y disminución del nivel de consciencia ${ }^{37}$. Un $72 \%$ de todas las HIE se presentan con coma, un $60 \%$ de los pacientes no comatosos muestra hemiplejia y un $43 \%$ alteraciones del lenguaje. Un $60 \%$ tienen cefalea antes, durante o después de la instauración del déficit neurológico, dato este que ayuda a diferenciar esta forma de ictus del isquémico. El déficit neurológico es progresivo en un 50-60\% de los pacientes y en la mayoría de ellos es debido al crecimiento del hematoma ${ }^{5,9,35,37}$. El crecimiento del hematoma en más de un $33 \%$ de su volumen se ha reportado en un $26 \%$ de los pacientes en la primera hora y en $12 \%$ adicional entre la $1^{\mathrm{a}}$ y la $20^{\mathrm{a}}$ horas. Este crecimiento puede verse agravado por la hipertensión arterial o trastornos de la coagulación ${ }^{5,10,35}$.

El diagnóstico de los hematomas en la HIE se fundamenta en la TAC urgente, técnica idónea para definir la naturaleza hemorrágica del ictus, e identificar la localización y el tamaño del hematoma. La arteriografía cerebral tiene un papel importante en pacientes menores de 45 años que pueden ser considerados candidatos al tratamiento quirúrgico y no presentan una causa clara para la hemorragia, si bien su rendimiento diagnóstico es mucho menor en los de mayor edad que no muestran anomalías sugestivas de lesiones subyacentes en la $\mathrm{TAC}^{5}$. La RM y angio-RM se han revelado como técnicas de gran sensibilidad en la detección de las lesiones asociadas a los hematomas intraparenquimatosos, pero su utilidad en el contexto de la atención urgente y la decisión quirúrgica en los pacientes con HIE está aún por definir. Conforme estas técnicas se realizan con mayor frecuencia se presta más atención al hecho de que en los pacientes afectos de HIE existen signos en RM de múltiples sangrados "menores" previos $5,9,10,37$.

A pesar de la alta incidencia (dobla la de la HSA en estudios poblacionales ${ }^{9}$ ) y de ser el proceso ictal con más alta mortalidad y morbilidad, el número de estudios encaminados a determinar su manejo óptimo es muy inferior a los dedicados a la HSA o la isquemia cerebral. Aún no se han realizado ensayos clínicos que hayan proporcionado evidencia científica definitiva sobre el beneficio real del tratamiento quirúrgico, que estaría teóricamente encaminado a disminuir el efecto masa, prevenir el resangrado y reducir la posible liberación de productos tóxicos desde el hematoma ${ }^{5,12,24,27,31,35,40}$. La hipertensión intracraneal debida al efecto masa del hematoma, el edema asociado y la potencial presencia de hidrocefalia continúan siendo la principal causa de muerte en los primeros días tras el ictus ${ }^{35}$. Aún cuando muchos aspectos relacionados con la etiopatogenia y la fisiopatología de la HIE están aún por determinar, el punto de mayor controversia e interés clínico sigue siendo el de su tratamiento quirúrgico.

El presente trabajo pretende revisar la literatura a fin de determinar las indicaciones de la evacuación quirúrgica en la HIE primaria supratentorial, también extrapolables a las hemorragias secundarias que no asocian una lesión cerebral subyacente. Además se discutirán los datos que han llevado a establecer dichas indicaciones para conocer el grado de certeza que las sostiene.

\section{Material y métodos}

En esta revisión se estudiaron los datos derivados de ensayos clínicos y meta-análisis respecto a la utilidad del tratamiento quirúrgico en el manejo de los hematomas intraparenquimatosos espontáneos supratentoriales. Para ello se emplearon preferentemente tanto los artículos hallados en la búsqueda en la base de datos Pub-Med como los artículos obtenidos de la revisión de la bibliografía de los primeros. Los términos empleados en la búsqueda fueron: "intracerebral hematoma" e "intracerebral hemorrhage" combinados con "surgery", "treatment" y "management".

\section{Resultados}

Se encontraron 10 ensayos clínicos y 5 meta-análisis en lengua inglesa que analizaban la utilidad del tratamiento quirúrgico en la HIE supratentorial (Tabla 2). La práctica totalidad de los estudios comparaban los resultados del tratamiento exclusivamente médico con los del tratamiento quirúrgico asociado al mejor tratamiento médico. Para ello evaluaron el porcentaje de pacientes que presentan una buena o mala evolución de acuerdo a la Escala de 
Tabla 2

Ensayos y meta-análisis en lengua inglesa sobre el tratamiento quirúrgico de la hemorragia intracerebral espontánea

\begin{tabular}{|c|c|c|}
\hline Autores & $A \tilde{\boldsymbol{n}} \boldsymbol{o}$ & $N^{o}$ de pacientes \\
\hline McKissock et $\mathrm{al}^{22}$ & 1961 & 180 \\
\hline Juvela et al. ${ }^{15}$ & 1989 & 52 \\
\hline Auer et al. ${ }^{1}$ & 1989 & 100 \\
\hline 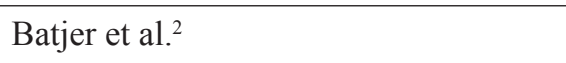 & 1990 & 21 \\
\hline Prasad et al. ${ }^{32}$ & 1997 & 353 \\
\hline Hankey et al ${ }^{11}$ & 1997 & 349 \\
\hline Morgenstern et al. ${ }^{24}$ & 1998 & 35 \\
\hline Zuccarello et al. ${ }^{43}$ & 1999 & 20 \\
\hline Prasad et al (Colaboración Cochrane) $)^{33}$ & 2000 & 354 \\
\hline Fernándes et al. ${ }^{8}$ & 2000 & 530 \\
\hline Teernstra et al. ${ }^{39}$ & 2003 & 71 \\
\hline Hattori $\mathrm{N}$ et al. ${ }^{12}$ & 2004 & 242 \\
\hline Mendelow et al. ${ }^{23}$ & 2005 & 1033 \\
\hline Teernstra et al. ${ }^{40}$ & 2006 & 1258 \\
\hline Pantazis et al. ${ }^{28}$ & 2006 & 108 \\
\hline
\end{tabular}

Evolución de Glasgow (GOS) (grados 4-5, o pacientes con "buena evolución", frente a los que presentan grados 1-3, o mala evolución con muerte o discapacidad grave). En uno de los estudios más recientes de los revisados ${ }^{23}$ se empleó la Escala Ampliada de Evolución de Glasgow (GOSE) y existieron dos umbrales diferentes de buena evolución en función del pronóstico de los pacientes al ingreso. Dos de los trabajos que estudiaron el efecto de la evacuación estereotáxica de los hematomas emplearon la Escala de Rankin Modificada como medida de la evolución funcional ${ }^{12,39}$. Los resultados de estos trabajos se resumen a continuación.

En 1961 McKissock et al. ${ }^{22}$, compararon la mortalidad y morbilidad en pacientes con HIE tratados médicamente o mediante evacuación por craneotomía del hematoma. El estudio englobó 180 pacientes con hematomas supratentoriales, tanto lobares como profundos, de los que 91 se incluyeron en el grupo de tratamiento médico y 89 en el grupo quirúrgico, analizándose los resultados según intención de tratamiento y comparando la evolución a los 6 meses. El resultado fue que la cirugía no aportaba beneficio respecto al tratamiento médico. La proporción de pacientes con mala evolución fue mayor en el grupo quirúrgico (casi
$80 \%$ ), que en el de tratamiento médico $(66 \%)$. En la mayoría de los pacientes la intervención se llevó a cabo 24 horas o más después del inicio de la clínica, y las imprecisiones en el proceso diagnóstico y la planificación del tratamiento conllevaron sesgos de selección hacia los casos más graves en el estudio (dada la ausencia de TAC, el diagnóstico se basó en los datos clínicos, arteriográficos y el resultado de la punción lumbar). Los pacientes alojados en el brazo quirúrgico, debían ser sometidos a ventriculografía para localizar el hematoma, interviniéndose aquellos "accesibles" quirúrgicamente (básicamente sólo aquéllos con hematomas lobares). De este modo, no todos los pacientes destinados a operarse recibieron dicho tratamiento, y en los operados, la precisión en la localización del hematoma estuvo limitada por la metodología diagnóstica.

En otro ensayo Juvela et al. ${ }^{15}$, compararon el tratamiento médico con el quirúrgico (craneotomía y evacuación del hematoma) en 52 pacientes ( 26 en cada brazo) con hematomas subcorticales y de ganglios basales. No se encontraron diferencias significativas en la proporción de fallecidos o pacientes dependientes, si bien los grupos eran desiguales en cuanto a factores pronósticos previos al tratamiento entre ambos grupos. Los pacientes trata- 
dos quirúrgicamente presentaron al ingreso una puntuación o "score" en la escala de coma de Glasgow (GCS) significativamente peor, y mostraron con mayor frecuencia hemorragia intraventricular, en tanto que los manejados médicamente tuvieron una proporción significativamente mayor de hematomas de ganglios basales. La conclusión del estudio fue que las HIE supratentoriales debían ser tratadas conservadoramente. Sin embargo, tras estratificar por la puntuación según la GCS al ingreso, se observó que si bien la evolución de los pacientes con puntuaciones extremadamente altas o bajas fue similar con los dos tipos de tratamiento, en aquellos con GCS entre 7 y 10 el tratamiento quirúrgico incrementó la supervivencia en comparación con el conservador de forma significativa. Por ello los autores consideraron que posibles estudios prospectivos futuros deberían enfocarse sobre este subgrupo de pacientes.

Otro estudio conducido por Batjer et al. ${ }^{2}$, trató de comparar el resultado del tratamiento médico convencional por un lado, el tratamiento médico con monitorización de PIC por otro, y el tratamiento quirúrgico (craneotomía y evacuación microquirúrgica a través de un abordaje transilviano) en pacientes con hematomas putaminales mayores de $3 \mathrm{~cm}$, excluyendo a los pacientes comatosos y a aquéllos que se encontraban alerta y con déficit leve. El estudio fue interrumpido debido a una evolución inesperadamente mala en los tres grupos de tratamiento, por lo que el número de pacientes resultó muy reducido para establecer una comparación estadística (21 en total). La proporción de pacientes con mala evolución fue del 85\% (11/13) en los grupos de tratamiento médico, y del $75 \%(6 / 8)$ en los intervenidos quirúrgicamente.

Auer et al. ${ }^{1}$, compararon los resultados del tratamiento en 100 pacientes con HIE de los que 50 fueron tratados médicamente y los otros 50 con evacuación del hematoma utilizando técnica endoscópica guiada por ecografía y dejando un drenaje en el lecho del hematoma durante unos días. Globalmente registraron una supervivencia y una probabilidad de buena evolución significativamente mejores en el grupo de pacientes tratados quirúrgicamente que en los no operados cuando ambas variables (supervivencia de un lado e independencia de otro) se evaluaban de forma independiente. La supervivencia a los 6 meses en los pacientes operados fue del $58 \%$, frente a un $30 \%$ en los pacientes que únicamente recibieron tratamiento médico. En los pacientes con hematomas de gran tamaño (mayores de 50 cc) el tratamiento quirúrgico no cambió significativamente la evolución funcional, pero sí mejoró la supervivencia. En los pacientes con hematomas pequeños la evacuación del hematoma mejoró el estado funcional, si bien la mortalidad fue similar a la registrada en el subgrupo sometido a tratamiento médico. El beneficio del tratamiento quirúrgico pareció restringirse a pacientes menores de 60 años y con hematomas lobares, aunque el escaso número de casos en cada subgrupo impidió obtener una conclusión definitiva.

Estos estudios fueron objeto de un meta-análisis en $1997^{32}$, en el que se compararon por separado los resultados de la craneotomía y la evacuación endoscópica de los hematomas con los observados tras tratamiento médico. Dicho análisis concluyó que la craneotomía aumentaba en un 13\% (Intervalo de Confianza al 95\%[IC 95\%] $=3.5 \%$ $23 \%$ ) el riesgo de mala evolución frente al tratamiento médico, si bien se reconocía que la interpretación de este resultado quedaba muy limitada por el peso relativo del mayor número de pacientes incluidos en el trabajo de McKissock et $\mathrm{al}^{22}$, en el cual se dieron diversas limitaciones (diagnóstico erróneo, sesgo de selección hacia pacientes más graves, y dificultades y retraso en el tratamiento quirúrgico debido a los problemas diagnósticos). En relación con la evacuación endoscópica, y reanalizando los resultados de estudio de Auer et al. ${ }^{1}$, se estimó que la cirugía aumentaba en un 18\% las probabilidades de buena evolución (IC 95\% entre un 36\% de reducción del riesgo de mala evolución y un $3.6 \%$ de incremento). Al realizar el análisis por subgrupos se hallaron resultados contradictorios con los referidos en el estudio original atribuibles a las diferencias con las pruebas estadísticas empleadas en el mismo; así, mientras parecía que el efecto beneficioso de la cirugía se limitaba efectivamente a los pacientes menores de 60 años de edad, dicho beneficio parecía independiente de la localización del hematoma por un lado, y mayor en los hematomas mayores de $50 \mathrm{cc}$ por otro.

Otro meta-análisis posterior ${ }^{11}$ sobre los mismos estudios concluyó que los pacientes sometidos a cirugía mostraban una tendencia no significativa a una peor evolución, con una Odds Ratio (OR) de mortalidad o dependencia para el tratamiento quirúrgico de 1.29 (IC 95\%=0.77-1.98), y para la craneotomía en particular de 2.10 (IC 95\%= 1.07-4.12). Además se revisaron de forma conjunta series de casos en las que tampoco se encontró beneficio con el tratamiento quirúrgico de los hematomas supratentoriales.

La Colaboración Cochrane ${ }^{33}$ utilizó así mismo los resultados de estos cuatro estudios para evaluar el papel de la cirugía en la HIE, concluyendo que no existe evidencia científica que confirme ni el beneficio, ni la mayor morbilidad asociadas al manejo quirúrgico. Se encontró una tendencia no significativa a una mayor probabilidad de muerte/dependencia con el tratamiento mediante craneotomía, reconociendo, sin embargo, la posible distorsión causada por el trabajo de McKissock et al. ${ }^{22}$, y la limitación que esto implicaba a la hora de extraer conclusiones válidas. De la misma manera, a pesar de encontrar una mejoría significativa de la mortalidad y una tendencia no significativa hacia una menor probabilidad de mortalidad/dependencia con la evacuación mediante cirugía endoscópica, se consideró que los resultados del estudio de Auer et al. ${ }^{1}$, debían 
ser interpretados con cautela debido al escaso número de pacientes. En esta revisión se remarcaba el hecho de que no existían además estudios que aclarasen el papel del tratamiento quirúrgico en los pacientes que experimentan un deterioro rápido del nivel de conciencia, los cuales se han considerado tradicionalmente los principales beneficiarios de la evacuación del hematoma.

En un ensayo piloto $^{24}$ en el que se compararon el tratamiento mediante craneotomía evacuadora del hematoma frente al tratamiento médico en un solo centro, tampoco se encontraron diferencias significativas en cuanto a la supervivencia, ni la proporción de pacientes con buena recuperación funcional, si bien existía una menor probabilidad de mala evolución en el grupo de pacientes operados $(69 \%$ de mala evolución en los pacientes que recibieron sólo tratamiento médico, frente a $50 \%$ en el grupo del tratamiento quirúrgico, $p=0.47$ ). Sin embargo, los grupos no eran comparables debido a una mayor proporción de hematomas profundos en el de los pacientes tratados quirúrgicamente.

En otro ensayo piloto también reciente, realizado por Zuccarello et al. ${ }^{43}$, en el que se compararon los resultados en pacientes tratados bien médicamente, o bien mediante cirugía (craneotomía o evacuación estereotáxica), tampoco se encontraron diferencias significativas en la mortalidad o el grado de dependencia a pesar de observarse una mayor probabilidad de buena evolución en los pacientes tratados quirúrgicamente $(\mathrm{OR}=0.48$, IC $95 \%=0.09-2.69)$ en la evaluación a los tres meses. En el grupo del tratamiento quirúrgico se observó una evolución significativamente mejor según la escala NIHSS (National Institutes of Health Stroke Scale). Se incluyeron 20 pacientes de los que 11 se manejaron con tratamiento médico sólo o con drenaje de LCR ventricular, y 9 con evacuación del hematoma (5 mediante craneotomía y 4 con evacuación estereotáxica y aplicación de fibrinolíticos a través de un drenaje en el lecho quirúrgico). En este estudio los grupos se encontraban desequilibrados en cuanto a factores pronósticos debido a una mayor proporción de hematomas profundos en los pacientes tratados médicamente.

Una de las revisiones más amplias y sistemáticas realizadas hasta la actualidad con el fin de elaborar guías clínicas para el manejo de los hematomas intraparenquimatosos espontáneos fue llevada a cabo por el grupo de trabajo reunido por la American Heart Association (AHA), cuyos resultados se publicaron en $1999^{5}$. De la revisión de los ensayos anteriores y de las numerosas series retrospectivas que evaluaron la eficacia del tratamiento quirúrgico en la HIE, únicamente pudieron concluir que parece recomendable el tratamiento conservador en los pacientes con hematomas menores de 10 cc, o déficit neurológico mínimo, mientras que los pacientes jóvenes con hematomas lobares de gran tamaño $(>50 \mathrm{cc}$ ) que sufren deterioro del nivel de consciencia son candidatos al tratamiento quirúrgico. Por otro lado, establecieron que en pacientes con $\mathrm{GCS} \leq 4$ este tratamiento no estaría indicado. Estas recomendaciones seleccionaban básicamente para el tratamiento quirúrgico a aquellos pacientes que tienen mayor probabilidad de mejorar y menor riesgo de empeorar con el mismo, y en los que no cabe otra medida terapéutica que la cirugía asumiendo como causa del deterioro la hipertensión intracraneal, o la compresión del sistema nervioso central sano.

En el año 2000 Fernandes et al. ${ }^{8}$ realizaron un meta-análisis incluyendo los seis estudios aleatorizados realizados hasta entonces. Se incluyó también otro ensayo realizado en China y reportado en el idioma natal que incluía 127 pacientes, de los que 64 recibieron tratamiento quirúrgico y 63 tratamiento médico ${ }^{6}$. En este último trabajo quedaban mal definidos los criterios de inclusión, se evaluaban conjuntamente pacientes con hematomas cerebelosos y supratentoriales, y existían diferencias significativas pretratamiento entre ambos brazos en cuanto al nivel de consciencia, volumen del hematoma y gravedad del déficit motor. Tras reconocer las limitaciones metodológicas de este trabajo y del estudio de McKissock et al. ${ }^{22}$ los autores realizaron dos análisis. En uno incluyeron los resultados de todos los estudios con aleatorización disponibles hasta la actualidad y en otro excluyeron los ensayos de McKissock et al. ${ }^{22}$ y el mencionado realizado en China. En tanto que la OR de mala evolución para la cirugía fue de 1.20 (IC 95\%, 0.83-1.74) cuando el análisis incluyó todos los estudios, disminuyó a 0.94 (IC 95\%, 0.60-1.47) cuando se excluyó el trabajo de McKissock et al. ${ }^{22}$, (19) y a 0.63 (CI 95\%, $0.35-1.14$ ) cuando se excluyeron éste y el de origen chino. Los autores del meta-análisis concluyeron que la cirugía mostraría una tendencia a la protección, si bien el beneficio comparativo con el tratamiento conservador no alcanzaría una diferencia estadísticamente significativa.

En 2003, y movidos por algunos trabajos que sugerían un beneficio de la evacuación estereotáxica de los hematomas con ayuda de fibrinolisis, Teernstra et al. ${ }^{39}$, llevaron a cabo un ensayo multicéntrico en el que se estudiaron 71 pacientes. Incluyeron a aquéllos de más de 45 años de edad, con hematomas de más de 10 cc y con cualquier puntuación en la escala de Glasgow, siendo necesaria para la inclusión la realización de la cirugía en las primeras 72 horas post-ictus. El tratamiento quirúrgico consistió en la implantación estereotáxica de un catéter en el centro del hematoma con aspiración de éste, y la posterior instilación cada 6 horas de dosis de 5000 UI de urokinasa durante un período de 48 horas. Cada instilación de urokinasa se sucedió de la aspiración del hematoma lisado a través del catéter. Un total de 36 pacientes recibieron tratamiento quirúrgico, y otros 35 se manejaron mediante tratamiento médico. Se analizó la supervivencia y la evolución de acuerdo a la Escala de Rankin Modificada a los 6 meses. Como variable secundaria se estudió también la reducción del volumen del 
hematoma. Los autores encontraron diferencias significativas pretratamiento entre ambos grupos en cuanto a antecedentes de patología cardiovascular, grado de deterioro neurológico y tamaño del hematoma (peores en el brazo quirúrgico), y los pacientes que recibieron tratamiento médico presentaron con mayor frecuencia hematomas profundos o sangrado intraventricular. A los 6 meses la mortalidad en el grupo quirúrgico fue de $56 \%$, frente a $59 \%$ en el grupo de tratamiento médico, sin diferencias significativas $(p=0,7)$, y tampoco se encontraron diferencias en cuanto al riesgo combinado de muerte y mala evolución neurológica $(\mathrm{OR}=0,52, \mathrm{p}=0,38)$. El tratamiento quirúrgico mostró sin embargo una reducción media del tamaño de los hematomas significativamente mayor que el tratamiento médico en las TAC de control a los 3 y 7 días.

En otro ensayo realizado en Japón y publicado en $2003^{12}$ se aleatorizaron 242 pacientes con hematomas putaminales en dos grupos de tratamiento de igual tamaño, unos manejados mediante tratamiento médico y otros mediante aspiración estereotáxica del hematoma. Estos pacientes fueron seleccionados de entre un grupo de 490 pacientes del que se excluyeron aquéllos que se encontraban completamente alerta o aquéllos que no presentaban apertura ocular al dolor. Se incluyeron pacientes de entre 35 y 85 años en los que el tratamiento pudiera ser realizado en menos de 24 horas desde el comienzo del cuadro. Se evaluó la evolución a un año, comparando la mortalidad y la recuperación funcional mediante la Escala de Rankin Modificada, agrupando a los pacientes en dependientes e independientes funcionalmente. Para la comparación se dividió a los pacientes entre los que presentaban apertura ocular ante estímulos leves y los que sólo lo hacían ante estímulos intensos. Entre los pacientes que precisaban estímulos intensos para la apertura ocular fallecieron un $11,8 \%$ de los operados frente a un $23,5 \%$ de los tratados médicamente, diferencia que fue estadísticamente significativa. El número de estos pacientes que presentó independencia funcional al año de evolución fue también significativamente mayor entre los operados $(47,1 \%)$ que entre los no operados $(21,6 \%)$. Entre los pacientes que presentaban apertura ocular ante estímulos leves se observó una tendencia no significativa a una menor mortalidad $(4,3 \%$ frente a $11,4 \%)$ y a una mayor probabilidad de buena evolución ( $52,9 \%$ frente a $40,0 \%$ ) entre los pacientes operados. En esta publicación no se ofrecieron los datos globales comparando la evolución del conjunto de pacientes operados con la de aquéllos no intervenidos.

Uno de los últimos ensayos realizados, el esperado $\mathrm{STICH}^{23}$, difiere de los realizados previamente en su planteamiento teórico de partida, ya que los pacientes sometidos a aleatorización no representaban al conjunto de los que padecían HIE, sino aquéllos en los que el cirujano dudaba de la indicación o no del tratamiento quirúrgico. En él se incluyeron 1033 pacientes, con edades entre 19 y 93 años y volúmenes del hematoma de entre 4 y $210 \mathrm{cc}$. Del total de pacientes, 965 pudieron ser sometidos a análisis, 529 asignados al tratamiento médico y 496 al brazo quirúrgico. No se encontraron diferencias significativas en cuanto a la supervivencia o la evolución neurológica a los 6 meses. La probabilidad de una evolución favorable fue de $26 \%$ para los pacientes asignados al tratamiento quirúrgico frente a un $24 \%$ para los asignados al tratamiento médico $(\mathrm{OR}=0.89$, $\mathrm{p}=0.414)$. Del análisis por subgrupos, aún reconociendo las limitaciones estadísticas del mismo, se observó que los pacientes con hematomas que llegaban a $1 \mathrm{~cm}$ o menos de la superficie cortical presentaban una tendencia a una mejor evolución con tratamiento quirúrgico que médico, siendo la interacción entre la profundidad del hematoma y el tipo de tratamiento significativa. En cuanto a las técnicas quirúrgicas la craneotomía mostró una tendencia no significativa a ofrecer mejores resultados que otros procedimientos. De forma llamativa aquellos pacientes con GCS $\leq 8$ mostraron una evolución uniformemente nefasta independientemente del tratamiento recibido ( $92 \%$ de mala evolución con cirugía y $84 \%$ con tratamiento médico). Cabe señalar que 140 de los 529 inicialmente asignados al brazo de tratamiento médico fueron posteriormente operados, la mayoría de ellos por deterioro clínico o radiológico (el análisis de los resultados se hizo por intención de tratamiento, y no se encontró que este transvase de pacientes influyera en los resultados, al presentar este pequeño grupo de pacientes una evolución similar a los demás, con un $22 \%$ de evolución favorable). También es importante reconocer que a pesar de que el estudio pretendió evaluar la utilidad del tratamiento quirúrgico precoz, se incluyeron pacientes dentro de las 72 horas de evolución, y existió un margen adicional de hasta 24 horas desde la aleatorización hasta la cirugía. Los propios autores reconocieron en este trabajo su impotencia para responder a la pregunta de si está justificado o no el tratamiento quirúrgico precoz de los hematomas cerebrales espontáneos así como para aclarar en qué circunstancias puede ser beneficioso. Ellos mismos afirmaron que cuando existen dudas de la indicación quirúrgica pueden estar justificadas, ya que la evolución probablemente es indiferente del tratamiento empleado.

En un meta-análisis reciente se incluyeron los 1258 pacientes de todos los estudios previos ${ }^{40}$. En tanto que el análisis global encontró una tendencia no significativa a una mejor evolución para la cirugía (OR de muerte: 0,84 , IC 95\%: 0,67-1,07; OR de mala evolución: 0,82, IC 95\%: 0,63-1,06) (de forma similar al estudio de Fernandes ${ }^{8}$ ), cuando se incluyeron únicamente aquellos ensayos con una adecuada calidad metodológica se encontró un efecto beneficioso y significativo para la cirugía (OR para muerte: 0,29, CI 95\%: 0,14-0,59; OR para mala evolución: 0,48, CI 95\%:0,24-0,96). En este último análisis la principal 
diferencia con el meta-análisis anterior fue la exclusión del ensayo de Juvela ${ }^{15}$, en el que los grupos de tratamiento presentaban diferencias significativas en cuanto a factores pronósticos pre-tratamiento. Se realizó también un análisis por subgrupos que no mostró diferencias significativas en la probabilidad de muerte o mala evolución para los pacientes intervenidos mediante craneotomía frente a los no operados, en tanto que observó una disminución significativa de ambas probabilidades para los pacientes intervenidos mediante evacuación estereotáxica del hematoma (OR de mortalidad: 0,29, IC 95\%. 0,14-0,59, OR de mala evolución: 0,48, IC 95\%: 0,24-0,96). Se debe tener en cuenta sin embargo que en el análisis por subgrupos no se incluyeron los resultados del $\mathrm{STICH}^{23}$ dado que no se pudieron analizar por separado los pacientes tratados mediante evacuación estereotáxica.

Por último, Pantazis et al. ${ }^{28}$ aleatorizaron 108 pacientes con hematomas subcorticales y putaminales en un estudio publicado durante la redacción de esta revisión. Los criterios de inclusión exigían que estos pacientes sufrieran hematomas de más de $30 \mathrm{cc}$ de volumen con afección del nivel de consciencia o de la función neurológica, y cuyo tratamiento pudiera ser realizado en menos de 8 horas. Se incluyeron 54 pacientes en cada grupo de tratamiento, y el objetivo principal fue comparar la supervivencia $\mathrm{y}$ evolución funcional al año del tratamiento. En tanto que la mortalidad a un año no fue significativamente diferente entre ambos grupos de tratamiento (48\% en el grupo quirúrgico frente a un $57 \%$ en el grupo conservador, $\mathrm{p}=0,337$ ), el porcentaje de pacientes con buena evolución fue significativamente mejor para los pacientes operados ( $33 \%$ frente a $9 \%, p=0,002)$. En el análisis por subgrupos tampoco se observaron diferencias significativas en cuanto a la mortalidad, aunque persistió un efecto beneficioso en cuanto a la proporción de pacientes con buena recuperación funcional como se detalla a continuación: en lo tocante al nivel de consciencia se observó que en pacientes con puntuación GCS entre 3 y 7 la cirugía no aportaba un beneficio significativo, en tanto que en aquéllos con una puntuación entre 8 y 14 el porcentaje de buena evolución entre los operados era del $72 \%$ frente a $21 \%$ de los no operados ( $\mathrm{p}<0,001)$; en cuanto a la localización del hematoma la cirugía mostró un beneficio significativo en cuanto a la recuperación funcional tanto para los hematomas lobares como para los putaminales; con respecto al volumen del hematoma el beneficio de la cirugía se restringió a aquellos pacientes con hematomas de 30-80 cc, no aportando mejoría a los pacientes con hematomas mayores de $80 \mathrm{cc}$.

\section{Discusión}

La actitud hacia el tratamiento quirúrgico de la HIE supratentorial ha estado durante muchos años condicio- nada por el resultado del primer ensayo realizado. Éste, se realizó en un medio sociosanitario completamente diferente al actual, marcado por la ausencia de la técnica que hoy consideramos básica para el diagnóstico y la planificación del tratamiento en esta patología: la TAC. Así, podemos explicarnos que en $4,5 \%$ de los pacientes operados y $5,5 \%$ de los tratados conservadoramente ni siquiera sufrieran HIE supratentorial, sino infartos masivos, metástasis, hematomas de cerebelo... Si además tenemos en cuenta que en la localización de las lesiones se emplearon los datos arteriográficos o de ventriculografía, y consideramos que el papel de la evacuación quirúrgica se encuentra altamente condicionado al daño que entraña el propio abordaje, nos podemos imaginar las consecuencias derivadas de esta imprecisión diagnóstica. El peso de los resultados de Mckissock se extiende más allá de su propio estudio. Dado el escaso número de pacientes incluidos en los ensayos que le siguieron, su influencia fue muy alta en los meta-análisis que se realizaron posteriormente. Considerando las carencias de los estudios de que se disponía hasta hace poco, se creó una gran expectación en torno al estudio STICH. Sin embargo, el planteamiento de partida de este hacía muy difícil encontrar alguna diferencia entre el tratamiento médico y conservador. Así, siendo el principal criterio de inclusión la indecisión terapéutica, se puede pensar que los pacientes incluidos serían aquéllos con criterios pronósticos suficientemente adversos a la cirugía como para que el neurocirujano no decidiese la evacuación del hematoma en primera instancia. Por otro lado, siendo esta premisa tan susceptible a la variabilidad en los criterios quirúrgicos, probablemente se incluirían también pacientes a quienes de entrada la cirugía no podía ofrecer ningún beneficio, como reflejan los valores extremos del diámetro de los hematomas que presentaban algunos de los pacientes.

Como consecuencia de todo lo anterior, las conclusiones en cuanto a la utilidad del tratamiento quirúrgico no pueden ir mucho más allá de las elaboradas por la AHA en $1999^{5}$, a las cuales únicamente se pueden añadir matices:

- La edad en la cual se plantearía el límite para la indicación del tratamiento quirúrgico no ha sido establecida con claridad, aunque el límite varía entre los 60 y los 80 años según los estudios ${ }^{1,13,28,31}$. Para establecer dicho límite parecen más importantes la edad biológica y la situación funcional basal de cada paciente que la edad cronológica, así como los factores pronósticos presentes en cada caso que permitan aventurar cierta calidad de vida tras la intervención.

- El criterio tal vez más importante y objetivo para la decisión de la indicación quirúrgica es el nivel de consciencia. De la misma manera que existe acuerdo en no tratar a aquellos pacientes con buen nivel de consciencia (GCS $\geq 13-14)$ un deterioro neurológico grave influye 


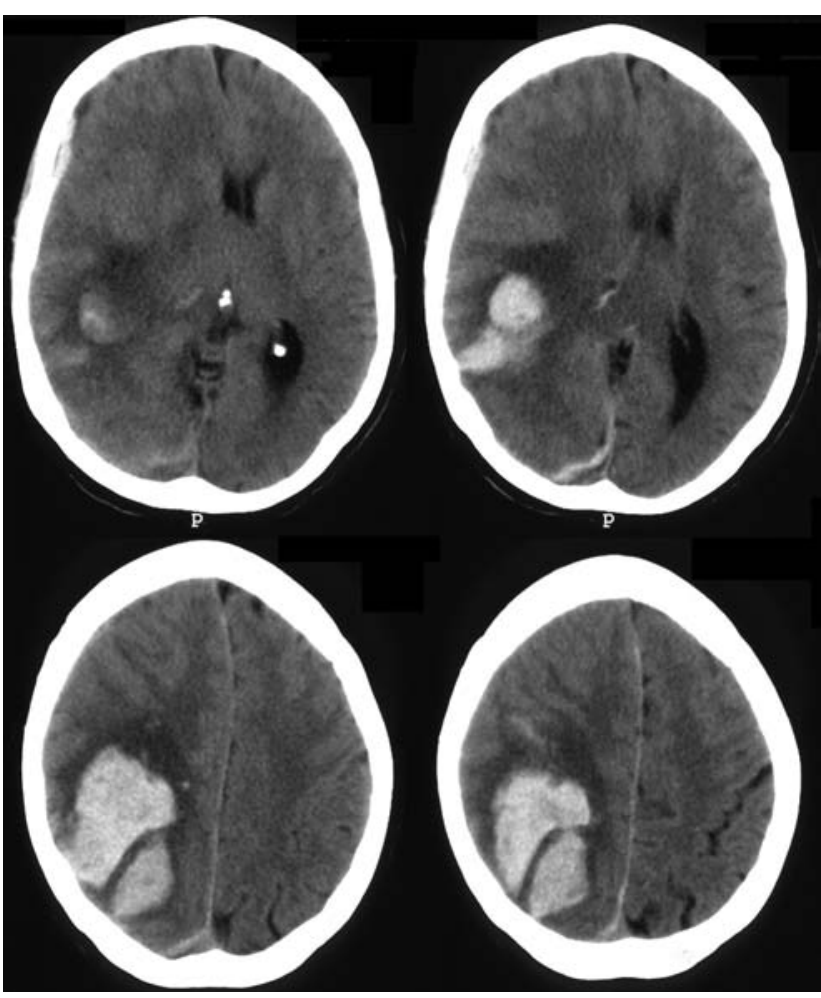

Figura 1. Mujer de 74 años, hipertensa, hipercolesterolémica e hipotiroidea. Fue llevada a la urgencia tras desarrollar hemiparesia izquierda, vómitos y progresiva disminución del nivel de consciencia. A su llegada a la urgencia presentaba apertura ocular y respuesta de localización con las extremidades derechas tras los estímulos dolorosos. Además se observó hemiplejia izquierda y midriasis derecha. La TAC mostró un hematoma de $6,5 \mathrm{~cm}$ de diámetro y $78 \mathrm{cc}$ de volumen. Fue intervenida mediante craneotomía y evacuación del hematoma y trasladada tras estabilización al servicio de rehabilitación. A los 60 dias del ictus presentaba adecuado nivel de consciencia, hemiparesia izquierda 4/5 y caminaba con ayuda. El estudio anatomopatológico del tejido resecado diagnosticó angiopatía amiloide.

significativamente en la ausencia de mejoría tras el tratamiento $5,9,14,16,27,31$. La evidencia derivada de trabajos experimentales y de estudios clínicos observacionales indica que los pacientes que parten de una situación clínica más grave tienen menor probabilidad de mejorar con el tratamiento quirúrgico ${ }^{4,14,17,30,36,41,42}$. El umbral crítico de afectación neurológica establecido por diferentes autores, por debajo del cual no estaría indicada la evacuación quirúrgica, varía, pero en general se establece en torno a una puntuación total de 4-5 en la GCS, estableciendo la mayoría de autores un $\mathrm{GCS}<5$ (respuesta motora $<3$ ) como criterio de exclusión para un tratamiento agresivo $0^{2,5,14,24,43}$. Parece claramente no indicada la cirugía en pacientes con respuesta de descerebración y ausencia de reflejos pontomesencefálicos ${ }^{27,36}$, y los datos derivados del STICH hacen pensar que con $\mathrm{GCS}<8$ la cirugía no aporta un beneficio significativo, dato similar al descrito por otros auto$\operatorname{res}^{15,23,28}$.

- En cuanto al requisito de que el hematoma sea voluminoso los hematomas de menos de $10 \mathrm{cc}$ presentan una evolución indiferente del tratamiento empleado, médico o quirúrgico, con lo que la cirugía no resulta beneficiosa o puede incluso ser perniciosa $a^{5,16}$. De modo inverso un volumen mayor de 50-60 cc se asocia a un peor pronóstico, y del reanálisis de los datos de Auer se concluyó que en el subgrupo de pacientes con hematomas de más de 50 cc la cirugía presentaba una tendencia a mejor evolución que el tratamiento médico ${ }^{5,24,33}$. Kanaya et al. en una serie amplia de hematomas putaminales establecieron en $30 \mathrm{cc}$ el umbral a partir del cual disminuye la mortalidad y mejora el pronóstico funcional respecto al tratamiento médico ${ }^{16}$, volumen límite también considerado por otros ${ }^{3,28}$.

- Aunque McKissock describió una peor evolución en los pacientes con hematomas localizados en el hemisferio dominante, no analizó la influencia que esto tenía en función del tratamiento seleccionado ${ }^{22}$. Otros autores han considerado este factor sin encontrar interacción entre la lateralidad del hematoma y el tipo de tratamiento, quirúrgico o conservador ${ }^{1,23}$, o bien han cuestionado la influencia de la alteración del lenguaje en el grado de recuperación funcional $^{9}$, o ni siquiera han considerado esta variable en sus recomendaciones ${ }^{8}$. La influencia de la lateralidad en la indicación del tratamiento quirúrgico es cuestionable aunque para algunos autores constituye un criterio más a valorar $^{31}$.

- En cuanto a la localización lobar o profunda, Auer describió una ausencia de beneficio con la cirugía en los hematomas profundos, pero estos hallazgos no se confirmaron en el reanálisis de sus datos ${ }^{1,32}$. Las guías de la AHA restringen su recomendación a los hematomas lobares ${ }^{5}$, aunque parece que más importante que el epicentro de la hemorragia puede ser su accesibilidad ${ }^{8}$, con una mejor evolución tras la cirugía de los pacientes con hematomas que alcanzan una distancia menor de $1 \mathrm{~cm}$ hasta la superficie cortical $^{23}$. Los hematomas putaminales representan una entidad más controvertida. En una serie retrospectiva de $7010^{16}$ pacientes se concluyó que la cirugía estaría indicada en hematomas putaminales de $>30$ cc que causan un deterioro significativo del nivel de consciencia. En los pacientes íntegros o desorientados el tratamiento médico fue mejor que el quirúrgico, en tanto que en el resto la cirugía consiguió disminuir la mortalidad aunque la evolución funcional no fue significativamente distinta en los supervivientes. En los pacientes definidos como semicomatosos sin signos de enclavamiento que fueron intervenidos la mortalidad fue del 30\%, frente al $60 \%$ de los manejados 


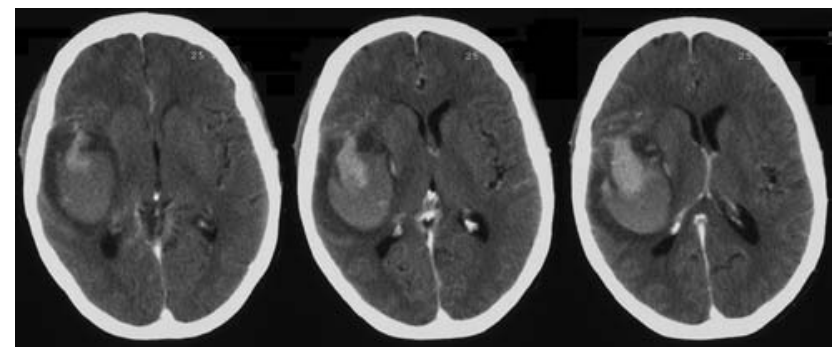

Figura 2. Paciente hepatópata de 41 años llevado a la urgencia por disminución del nivel de consciencia $y$ hemiparesia izquierda. Se encontraba somnoliento, desorientado, con hemiparesia izquierda de predominio braquial. La analítica mostró una importante pancitopenia y una actividad de protrombina de 46\%. La TAC diagnosticó un hematoma espontáneo de la cápsula externa con diferentes fases de sangrado. El diámetro máximo era de $5,6 \mathrm{~cm}$ y su volumen de $63 \mathrm{cc}$, con discreto desplazamiento de linea media. Fue tratado mediante craneotomía y evacuación del hematoma, siendo dado de alta hospitalaria a los 21 días, con deambulación independiente y leve paresia distal en el miembro superior izquierdo.

médicamente. En aquellos pacientes semicomatosos con signos de enclavamiento, la mortalidad con el tratamiento quirúrgico alcanzó el $60 \%$, y en aquéllos en coma profundo el $80 \%$. Teniendo en cuenta esto, en nuestra opinión los pacientes con $\mathrm{GCS} \leq 13$ y hematomas putaminales de más de $30 \mathrm{cc}$, deben ser intervenidos cuando presentan una respuesta motora mejor a la postura de decorticación, grupo que según el estudio anterior sería el máximo beneficiario de la evacuación quirúrgica al conseguir una mejora en la supervivencia. En los pacientes con peor situación neurológica, a pesar que la cirugía mejore la supervivencia las probabilidades de muerte serían demasiado altas ${ }^{16}$. Los resultados del reciente ensayo de Pantazis et al. sugieren también un beneficio de la cirugía en pacientes con deterioro neurológico secundario a hematomas putaminales de más de $30 \mathrm{cc}$, aunque también refieren una ausencia de mejoría con la cirugía cuando el deterioro es avanzado ${ }^{28}$. En cuanto a los hematomas talámicos parecen tener una evolución extremadamente pobre con cualquier tratamiento, y su tratamiento quirúrgico se considera prácticamente abandonado $^{5,9,27,28}$.

- La HIV se ha reconocido como uno de los factores más uniformemente asociado a mala evolución en la HIE. Su efecto pernicioso se podría deber tanto a la existencia de hidrocefalia secundaria, como al efecto masa ejercido sobre el tejido periventricular (con alteraciones locales del flujo sanguíneo) como a los efectos tóxicos de la sangre. Si bien clásicamente su manejo se basaba en la colocación de un drenaje ventricular externo, se ha reportado con frecuencia creciente el uso de fibrinolíticos administrados a través del mismo catéter. Este tratamiento parece reducir el tiempo de permanencia de la sangre en los ventrículos y disminuir la mortalidad de los pacientes en torno a un 30\%, aún cuando no está todavía claro si mejora la evolución neurológica en los supervivientes?

- En cuanto a la técnica quirúrgica a emplear para la evacuación del hematoma no hay datos que permitan seleccionar la craneotomía o procedimientos menos invasivos. En numerosas series de casos se ha recurrido al empleo de técnicas menos invasivas que la craneotomía para la evacuación de los hematomas intraparenquimatosos. Así, se ha utilizado la simple aspiración no guiada (con la consecuente dificultad para la localización del hematoma y su insuficiente evacuación) o bien procedimientos guiados por estereotaxia o ecografía intraoperatoria logrando evacuaciones que oscilan entre el 70 y $80 \%$ de la masa del hematoma ${ }^{19,26,39}$. La combinación de trombolisis con aspiración del hematoma por métodos estereotáxicos ha sido utilizada por diversos autores logrando evacuaciones de un 30-90\% del volumen del hematoma ${ }^{5}$. En tanto que los estudios de Auer et al y Hattori et al., mostraron un beneficio del tratamiento quirúrgico, el ensayo publicado por Teernstra et al., no encontró una mejor evolución clínica en los pacientes operados. Si la comparación de los resultados del tratamiento médico y quirúrgico de las HIE supratentoriales no arroja datos concluyentes, mucho menos lo hace la de los resultados obtenidos con las diferentes técnicas quirúrgicas, y a pesar de los datos a favor de técnicas menos invasivas el STICH muestra una tendencia no significativa a una mejor evolución con la craneotomía, que es a la postre la técnica más empleada ${ }^{5,23}$.

La ausencia de evidencia en este campo merece aún el desarrollo de ensayos clínicos que definan mejor el papel del tratamiento, no sólo quirúrgico, sino también médico. Este último hasta ahora no va más allá del mero soporte vital y tratamiento de la hipertensión intracraneal ${ }^{10,21} \mathrm{y}$ sin embargo están en marcha ensayos de tratamientos que podrían atajar una de las principales causas de deterioro en estos pacientes: el crecimiento del hematoma debido a un sangrado continuado o a resangrado. El factor VII recombinante activado, empleado en pacientes hemofílicos que sufren hemorragias, ha sido estudiado recientemente en tres dosis diferentes frente a placebo en un ensayo fase IIB $^{20}$, analizando como medida primaria el porcentaje de incremento en el volumen de hematoma y como medidas secundarias la supervivencia y evolución neurológica. Se aleatorizaron 400 pacientes (96 recibieron placebo, 108 pacientes recibieron $40 \mathrm{microg} / \mathrm{kg}$ de factor VII, 92 pacientes recibieron $80 \mathrm{microg} / \mathrm{kg}$ y 103 pacientes recibieron $160 \mathrm{microg} / \mathrm{kg}$ ). Los pacientes que recibieron factor VII mostraron una disminución significativa del crecimiento del hematoma cuando se analizaron conjuntamente los tres grupos de dosis. Analizando cada subgrupo sólo los pacientes 
que recibieron la dosis más alta de factor VII presentaron una disminución significativa en el crecimiento del hematoma respecto al placebo. Esto podría representar bien la ausencia de efecto a dosis menores o la imposibilidad para demostrar el mismo debido al reducido número de pacientes. En cuanto a la evolución clínica los pacientes que recibieron factor VII mostraron una mortalidad significativamente menor de forma global, aunque no por subgrupos. La evolución funcional fue significativamente mejor para todos los rangos de dosis de forma individual y también de forma global cuando se evaluó según las escalas de Rankin Modificada, NIHSS y Barthel, aunque no se encontró diferencia significativa empleando el GOSE (atribuido al efecto del gran número de pacientes en los grados de mortalidad y mayor discapacidad tanto en los grupos placebo como factor VII). El beneficio del tratamiento con este factor pareció limitarse a los pacientes que lo recibieron dentro de las primeras tres horas. Como contrapartida los pacientes tratados con factor VII mostraron un número significativamente mayor de eventos adversos atribuibles a tromboembolia, fundamentalmente arterial, tanto cerebral como cardíaca, con un riesgo casi triple que el de los pacientes que recibieron placebo. En conjunto los pacientes tratados con factor VII presentaron un riesgo de muerte o incapacidad grave un $16 \%$ menor, con un número necesario de pacientes a tratar de entre 6 y 7 para evitar un evento de mala evolución, de acuerdo a la escala modificada de Rankin. Los resultados de este trabajo son un paso muy inicial antes de su aplicación clínica extensiva, y el factor VII recombinante aún no ha sido aprobado por la FDA para su uso en esta patología. Queda aún por definir la dosis con un mejor balance entre el beneficio y los eventos adversos (160 microg/kg es casi el doble de la dosis empleada en los pacientes hemofílicos), así como el papel de este tratamiento en los pacientes con factores de riesgo tromboembólico o anticoagulados, que fueron excluidos del ensayo (representan una importante proporción del total de pacientes seguramente, si tenemos en cuenta los factores de riesgo tanto para la enfermedad cardiovascular como para la hemorragia intracerebral espontánea).

Merece un comentario aparte la HIE asociada al tratamiento anticoagulante. En torno a un $10-23 \%$ de pacientes con esta patología recibe anticoagulantes orales, que son al mismo tiempo factor de riesgo para su desarrollo (RR entre 7 y 10) y factor pronóstico adverso en cuanto a la evolución ${ }^{10,34,38}$. La intensidad de la anticoagulación es también al mismo tiempo factor de riesgo y un factor predictor independiente de mortalidad, empeorando ambos conforme mayor es el INR ${ }^{34,38}$. Aunque es controvertido el grado en que los anticoagulantes se relacionan con un mayor volumen del hematoma, sí se han correlacionado con un mayor riesgo de crecimiento del mismo. Ante la presencia de un HIE en estos pacientes se debe recurrir a la reversión de la anticoagulación lo más rápido posible, mediante la admi- nistración de vitamina $\mathrm{K}$ y factores de la coagulación, en forma de plasma fresco congelado o preparados comerciales de protrombina ${ }^{10,34,38}$.

\section{Conclusiones}

Considerando lo anteriormente expuesto parece claro que pacientes con HIE supratentorial y GCS 14-15 o hematomas menores de $3 \mathrm{~cm}$ de diámetro no deben ser intervenidos. Aquellos pacientes con GCS 6-13 y hematomas mayores de $3 \mathrm{~cm}$ de diámetro pueden verse beneficiados de la evacuación quirúrgica de los hematomas lobares y putaminales cuando por su edad y situación basal exista la posibilidad de cierta calidad de vida posterior. En pacientes con GCS $<5-6$ resulta muy difícil establecer una base para la indicación quirúrgica, que probablemente deba desestimarse cuando la respuesta motora sea peor a la postura de decorticación y se acompañe de alteración de los reflejos troncoencefálicos. Dado que no existe una evidencia firme que permita establecer unas indicaciones estrictas del tratamiento quirúrgico, éste se decidirá de un modo individualizado, y considerando el entorno socio-familiar de cada paciente. Dada la alta tasa de discapacidad entre los supervivientes, éste último factor resulta determinante en cuanto a las expectativas de recuperación y calidad de vida del paciente.

La ausencia de un beneficio claro de la cirugía en la HIE supratentorial en los ensayos realizados hasta ahora, puede deberse en parte a la inclusión de pacientes que de entrada presentaban escasas posibilidades de beneficiarse de este tratamiento. Por otro lado, la GOS y GOSE son escalas validadas en el ámbito del traumatismo craneoencefálico. $\mathrm{Su}$ empleo en el ictus hemorrágico puede enmascarar el beneficio de una medida terapéutica concreta, ya que la dicotomía buena/mala evolución puede resultar de escaso valor discriminatorio. Tal vez, si se desarrollasen futuros estudios, debieran emplearse escalas de evolución validadas en el ictus, tales como la Escala de Rankin Modificada.

\section{Bibliografía}

1. Auer, L.M., Deinsberger, W., Niederkorn, K., et al.: Endoscopic surgery versus medical treatment for spontaneous intracerebral hematoma: a randomized study. J Neurosurg 1989; 70: 530-535.

2. Batjer, H.H., Reisch, J.S., Allen, B.C., Plaizier, L.J., Su, C.J.: Failure of surgery to improve outcome in hypertensive putaminal hemorrhage. A prospective randomized trial. Arch Neurol 1990; 47: 1103-1106.

3. Bilbao, G., Garibi, J., Pomposo, I., et al.: A prospective study of a series of 356 patients with supratentorial spontaneous intracerebral haematomas treated in a Neurosurgical Department. Acta Neurochir (Wien) 2005; 147: 823-829. 
4. Brambilla, G.L., Baena, R., Sangiovanni, G., Rainoldi, F., Locatelli, D.: Spontaneous intracerebral hemorrhage: medical or surgical treatment. J Neurosurg Sci 1983; 27: 95101.

5. Broderick, J.P., Adams, H.P., Jr., Barsan, W., et al.: Guidelines for the management of spontaneous intracerebral hemorrhage: A statement for healthcare professionals from a special writing group of the Stroke Council, American Heart Association. Stroke 1999; 30: 905-915.

6. Chen, X., Yang, H., Czherig, Z.: A prospective randomised trial of surgical and conservative treatment in hypertensive intracranial hemorrhage. Acta Acad Med Shanghai 1992; 19: 237-240.

7. Engelhard, H.H., Andrews, C.O., Slavin, K.V., Charbel, F.T.: Current management of intraventricular hemorrhage. Surg Neurol 2003; 60: 15-21.

8. Fernandes, H.M., Gregson, B., Siddique, S., Mendelow, A.D.: Surgery in intracerebral hemorrhage. The uncertainty continues. Stroke 2000; 31: 2511-2516.

9. Fewel, M.E., Thompson, B.G., Jr., Hoff, J.T.: Spontaneous intracerebral hemorrhage: a review. Neurosurg Focus [Revista electrónica] 2003; 15(4): Artículo 1[16 p]. Disponible en: http://www.aans.org/education/journal/neurosurgical/ oct03/15-4-1.pdf.

10. Flaherty, M.L.: Anticoagulant-associated intracerebral hemorrhage. Issues in Hemostasis Management 2005; 1: 1-9.

11. Hankey, G.J., Hon, C.: Surgery for primary intracerebral hemorrhage: is it safe and effective? A systematic review of case series and randomized trials. Stroke 1997; 28: 21262132 .

12. Hattori, N., Katayama, Y., Maya, Y., Gatherer, A.: Impact of stereotactic hematoma evacuation on activities of daily living during the chronic period following spontaneous putaminal hemorrhage: a randomized study. J Neurosurg 2004; 101: 417-420.

13. Izumihara, A., Ishihara, T., Iwamoto, N., Yamashita, K., Ito, H.: Postoperative outcome of 37 patients with lobar intracerebral hemorrhage related to cerebral amyloid angiopathy. Stroke 1999; 30: 29-33.

14. Juvela, S.: Risk factors for impaired outcome after spontaneous intracerebral hemorrhage. Arch Neurol 1995; 52: $1193-1200$.

15. Juvela, S., Heiskanen, O., Poranen, A., et al.: The treatment of spontaneous intracerebral hemorrhage. A prospective randomized trial of surgical and conservative treatment. J Neurosurg 1989; 70: 755-758.

16. Kanaya, H., Kuroda, K.: Development in neurosurgical approaches to hypertensive intracerebral hemorrhage in Japan. En Kaufmann, H.H. (ed). Intracerebral Hematomas. New York; Raven Press Ltd, 1992; pp.197-210.

17. Kingman, T.A., Mendelow, A.D., Graham, D.I., Teasdale, G.M.: Experimental intracerebral mass: time-related effects on local cerebral blood flow. J Neurosurg 1987; 67:
732-738.

18. Lee, K.R., Kawai, N., Kim, S., Sagher, O., Hoff, J.T.: Mechanisms of edema formation after intracerebral hemorrhage: effects of thrombin on cerebral blood flow, blood-brain barrier permeability, and cell survival in a rat model. J Neurosurg 1997; 86: 272-278.

19. Matsumoto, K., Hondo, H.: CT-guided stereotaxic evacuation of hypertensive intracerebral hematomas. J Neurosurg 1984; 61: 440-448.

20. Mayer, S.A., Brun, N.C., Begtrup, K., et al.: Recombinant activated factor VII for acute intracerebral hemorrhage. $\mathrm{N}$ Engl J Med 2005; 352: 777-785.

21. Mayer, S.A., Rincon, F.: Treatment of intracerebral haemorrhage. Lancet Neurol 2005; 4: 662-672.

22. McKissock, W.: Primary intracerebral hemorrhage. A controlled trial of surgical and conservative treatment in 180 unselected cases. Lancet 1961; 2: 221-226.

23. Mendelow, A.D., Gregson, B.A., Fernandes, H.M., et al.: Early surgery versus initial conservative treatment in patients with spontaneous supratentorial intracerebral haematomas in the International Surgical Trial in Intracerebral Haemorrhage (STICH): a randomised trial. Lancet 2005; 365 : 387-397.

24. Morgenstern, L.B., Frankowski, R.F., Shedden, P., Pasteur, W., Grotta, J.C.: Surgical treatment for intracerebral hemorrhage (STICH): a single-center, randomized clinical trial. Neurology 1998; 51: 1359-1363.

25. Nath, F.P., Kelly, P.T., Jenkins, A., Mendelow, A.D., Graham, D.I., Teasdale, G.M.: Effects of experimental intracerebral hemorrhage on blood flow, capillary permeability, and histochemistry. J Neurosurg 1987; 66: 555-562.

26. Niizuma, H., Shimizu, Y., Yonemitsu, T., Nakasato, N., Suzuki, J.: Results of stereotactic aspiration in 175 cases of putaminal hemorrhage. Neurosurgery 1989; 24: 814-819.

27. Ojemann, R.G., Heros, R.C.: Spontaneous brain hemorrhage. Stroke 1983; 14: 468-475.

28. Pantazis, G., Tsitsopoulos, P., Mihas, C., Katsiva, V., Stavrianos, V., Zymaris, S.: Early surgical treatment vs conservative management for spontaneous supratentorial intracerebral hematomas: a prospective randomized study. Surg Neurol 2006; 66: 492-501.

29. Papo, I., Janny, P., Caruselli, G., Colnet, G., Luongo, A.: Intracranial pressure time course in primary intracerebral hemorrhage. Neurosurgery 1979; 4: 504-511.

30. Pia, H.W., Langamid, C., Zierski, J.: Spontaneous intracerebral hematomas. Advances in diagnosis and therapy. Berlin; Springer-Verlag 1980.

31. Pouratian, N., Kassell, N.F., Dumont, A.S.: Update on management of intracerebral hemorrhage. Neurosurg Focus [Revista electrónica] 2003; 15(4): Artículo 2[6 p]. Disponible en: http://www.aans.org/education/journal/neurosurgical/oct03/15-4-2.pdf.

32. Prasad, K., Browman, G., Srivastava, A., Menon, G.: 
Surgery in primary supratentorial intracerebral hematoma: a meta-analysis of randomized trials. Acta Neurol Scand 1997; 95: 103-110.

33. Prasad, K., Shrivastava, A.: Surgery for primary supratentorial intracerebral haemorrhage (Cochrane Review). En The Cochrane Lybrary, Volumen 1. Chichester, UK; John Wiley \& Sons, Ltd, 2004.

34. Quinones-Hinojosa, A., Gulati, M., Singh, V., Lawton, M.T.: Spontaneous intracerebral hemorrhage due to coagulation disorders. Neurosurg Focus [Revista electrónica] 2003; 15(4): Artículo 3 [17p]. Disponible en: http://www.aans.org/ education/journal/neurosurgical/oct03/15-4-3.pdf.

35. Qureshi, A.I., Tuhrim, S., Broderick, J.P., Batjer, H.H., Hondo, H., Hanley, D.F.: Spontaneous intracerebral hemorrhage. N Engl J Med 2001; 344: 1450-1460.

36. Rabinstein, A.A., Atkinson, J.L., Wijdicks, E.F.: Emergency craniotomy in patients worsening due to expanded cerebral hematoma: to what purpose? Neurology 2002; 58: 1367-1372.

37. Rodríguez Peña-Marín, M., Pérez-Núñez, A., Martinez-Salio, A., Lobato R.D.: Hemorragia intracraneal. En Gonzalez de la Aleja, J., Rodríguez Peña-Marín, M., Sepúlveda J. (ed). Urgencias en Neurología. Madrid; Jarpyo Editores, 2006; pp. 250-266.

38. Rosand, J., Eckman, M.H., Knudsen, K.A., Singer, D.E., Greenberg, S.M.: The effect of warfarin and intensity of anticoagulation on outcome of intracerebral hemorrhage. Arch Intern Med 2004; 164: 880-884.
39. Teernstra, O.P., Evers, S.M., Lodder, J., Leffers, P., Franke, C.L., Blaauw, G.: Stereotactic treatment of intracerebral hematoma by means of a plasminogen activator: a multicenter randomized controlled trial (SICHPA). Stroke 2003; 34: 968-974.

40. Teernstra, O.P., Evers, S.M., Kessels, A.H.: Meta analyses in treatment of spontaneous supratentorial intracerebral haematoma. Acta Neurochir (Wien ) 2006; 148: 521-528.

41. Volpin, L., Cervellini, P., Colombo, F., Zanusso, M., Benedetti, A.: Spontaneous intracerebral hematomas: a new proposal about the usefulness and limits of surgical treatment. Neurosurgery 1984; 15: 663-666.

42. Waga, S., Yamamoto, Y.: Hypertensive putaminal hemorrhage: treatment and results. Is surgical treatment superior to conservative one? Stroke 1983; 14: 480-485.

43. Zuccarello, M., Brott, T., Derex, L. et al.: Early surgical treatment for supratentorial intracerebral hemorrhage: a randomized feasibility study. Stroke 1999; 30: 1833-1839.

Pérez-Núñez, A.; Lagares, A.; Pascual, B.; Rivas, J.J.; Alday, R.; González, P.; Cabrera, A.; Lobato, R.D.: Tratamiento quirúrgico de la hemorragia intracerebral espontánea. Parte I: Hemorragia supratentorial. Neurocirugía 2008; 19: 12-24.

Correspondencia postal: Ángel Pérez Núñez. Servicio de Neurocirugía. Hospital Doce de Octubre. Avda de Córdoba s/n. 28041 Madrid 